\title{
Diabetic Ketoacidosis and
}

\section{Hyperosmolar Hyperglycemic Syndrome Management}

H. Evan Dingle, MD; Corey Slovis, MD

\section{In this review, the authors discuss the similarities and differences between diabetic ketoacidosis and the hyperosmolar hyperglycemic state, providing clinical pearls and common pitfalls to help guide the clinician in the diagnosis and management.}

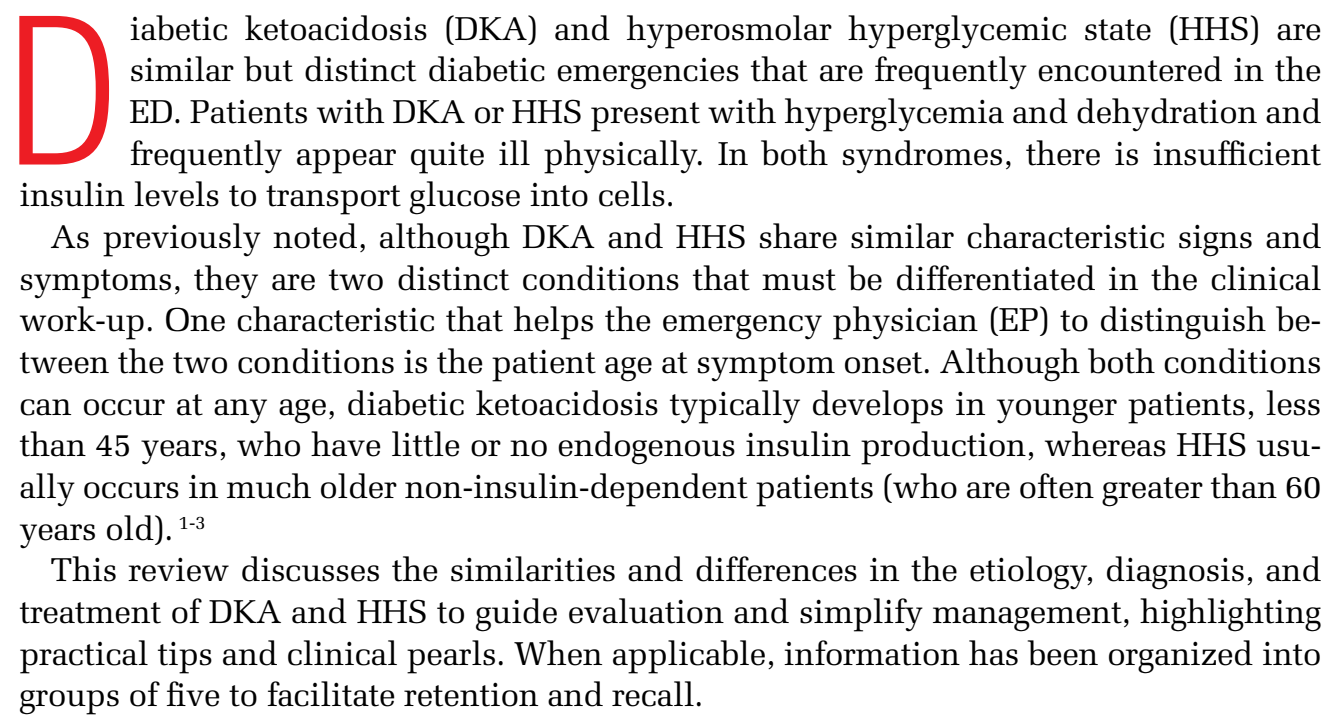

The Etiology of DKA Vs HHS

The fundamental underlying issue in both DKA and HHS is an absolute or relative lack of insulin that results in an increase in counter-regulatory hormones, including glucagon, cortisol, and catecholamines.

Insulin has five main actions: (1) to drive glucose into cells; (2) to drive potassium into

Dr Dingle is an assistant professor, department of emergency medicine, Vanderbilt University Medical Center, Nashville, Tennessee; medical director, Nashville Veterans Affairs' new emergency medical service, Tennessee; and an assistant medical director, Nashville Fire Department, Tennessee. Dr Slovis is chair, department of emergency medicine, Vanderbilt University Medical Center, Nashville, Tennessee; and medical director, Nashville Fire Department and Nashville International Airport, Tennessee.

Authors' Disclosure Statement: The authors report no actual or potential conflict of interest in relation to this article.

DOI: 10.12788/emed.2018.0100 


\section{DIABETIC KETOACIDOSIS AND HYPEROSMOLAR HYPERGLYCEMIC SYNDROME MANAGEMENT}

Table 1. Five Actions of Insulin for Diabetic Ketoacidosis (DKA) Treatment

\begin{tabular}{ll}
\hline Action & DKA Condition Treated \\
\hline Drives glucose into cells & Hyperglycemia \\
\hline Drives potassium into cells & Hyperkalemia \\
\hline Anabolic & Catabolism \\
\hline Blocks fat breakdown & Free fatty acid production \\
\hline Blocks protein breakdown & Ketoacid production \\
\hline
\end{tabular}

cells; (3) to create an anabolic environment; (4) to inhibit breakdown of fat; and (5) to block the breakdown of proteins. (Table 1).

Diabetic ketoacidosis typically develops in patients who lack significant endogenous insulin; this insufficiency of circulating insulin causes hyperglycemia and hyperkalemia, the creation of a catabolic state with high levels of both ketone bodies and free-fatty acids due to the breakdown of proteins and fats.

In contrast, HHS occurs in patients who produce a sufficient amount of insulin to drive potassium into cells and to inhibit the breakdown of proteins and fats; as such these patients are not ketoacidotic. However, patients with HHS do not produce enough insulin to drive glucose intracellularly. As the glucose levels increase, patients with HHS become increasingly hyperosmolar and dehydrated, resulting in further elevation of glucose levels, causing a perpetual cycle of increasing glucose and resultant hyperosmolarity and dehydration..$^{1-3}$ It is important to appreciate that both hyperglycemic crises result in an osmotic diuresis leading to severe dehydration and urinary wasting of electrolytes.

\section{Diagnosis and Workup}

\section{Laboratory Evaluation}

In addition to hyperglycemia, another key finding in DKA is elevated anion gap metabolic acidosis resulting from ketoacid production. Laboratory evaluation will demonstrate a pH less than 7.3 and serum bicarbonate level less than $18 \mathrm{mEq} / \mathrm{L}$; urinalysis will be positive for ketones.

Though HHS patients also present with hyperglycemia, laboratory evaluation will demonstrate no, or only mild, acidosis, normal $\mathrm{pH}$ (typically $>7.3$ ), bicarbonate level greater than $18 \mathrm{mEq} / \mathrm{L}$, and a high-serum osmolality (>350 mOsm $/ \mathrm{kg}$ ). While urinalysis may show low or no ketones, patients with HHS do not develop the marked ketoacidosis seen in DKA. Moreover, glucose values are more elevated in patients with HHS, frequently exceeding $1,000 \mathrm{mg} / \mathrm{dL}$.

\section{Signs and Symptoms}

Patients with either of these hyperglycemic crises often present with fatigue, polyuria, and polydipsia. They appear dehydrated on examination with dry mucous membranes, tachycardia, and in severe cases, hypotension. Patients with HHS often present with altered mental status, seizures, and even coma, while patients with DKA typically only experience changes in mental status in the most severe cases (Table 2). As many as onethird of patients with a hyperglycemic crisis will have an overlapping DKA/HHS syn- 


\section{DIABETIC KETOACIDOSIS AND HYPEROSMOLAR HYPERGLYCEMIC SYNDROME MANAGEMENT}

Table 2. Characteristics of Diabetic Ketoacidosis (DKA) and Hyperosmolar Hyperglycemic State (HHS)

\begin{tabular}{lll}
\hline & DKA & HHS \\
\hline Endogenous insulin levels & Very low & May be within normal limits \\
\hline Ketoacidosis & Profound & Minimal \\
\hline Glucose & $>250 \mathrm{mg} / \mathrm{dL}$ (often around $600 \mathrm{mg} / \mathrm{dL}$ ) & $>600 \mathrm{mg} / \mathrm{dL}$ (often $>1,000 \mathrm{mg} / \mathrm{dL}$ ) \\
\hline Serum bicarbonate & $5-15 \mathrm{mEq} / \mathrm{L}$ & $20-24 \mathrm{mEq} / \mathrm{L}$ \\
\hline Osmolality & $300-325$ mosm $/ \mathrm{kg}$ & $>350 \mathrm{mosm} / \mathrm{kg}$ \\
\hline Age & Younger (most $<45$ years) & Older (often $>60$ years) \\
\hline Onset & Acute & Subacute/Chronic \\
\hline Associated diseases & Rare & Common \\
\hline Seizures & Very rare & Common \\
\hline Coma & Rare & Common \\
\hline Mortality & Approaches 0 & $10 \%-20 \%$ \\
\hline
\end{tabular}

Table 3. The Five Causes of Diabetic Ketoacidosis and Hyperosmolar Hyperglycemic State (Five "I's")

\begin{tabular}{ll}
\hline Cause & Examples \\
\hline Infection & Urinary tract infection, pneumonia, cellulitis/soft tissue infection \\
\hline Infarction & Myocardial infarction, stroke, bowel, skin \\
\hline Indiscretion with sugar & Noncompliance with diabetic diet \\
\hline Insulin (lack of) & Skipping insulin doses, insulin pump failure \\
\hline Infant (pregnancy) & Pregnancy test should be given to all patients of child-bearing age \\
\hline
\end{tabular}

drome. ${ }^{1-3}$ With respect to patient age, HHS tends to develop in older patients who have type 2 diabetes and several underlying comorbidities. ${ }^{2}$

\section{Precipitating Causes of DKA and HHS}

The five causes of DKA/HHS can be remembered as the "Five I's": (1) infection; (2) infarction; (3) infant (pregnancy), (4) indiscretion, and (5) [lack of] insulin (Table 3).

Infection. Infection is one of the most common precipitating factors in both DKA and HHS. ${ }^{1}$ During the history intake, the EP should ask the patient if he or she has had any recent urinary and respiratory symptoms, as positive responses prompt further investigation with urinalysis and chest radiography. A thorough dermatological examination should be performed to assess for visible signs of infection, particularly in the extremities and intertriginous areas.

When assessing patients with DKA or HHS for signs of infection, body temperature to 


\section{DIABETIC KETOACIDOSIS AND HYPEROSMOLAR HYPERGLYCEMIC SYNDROME MANAGEMENT}

assess for the presence of fever is not always a reliable indicator. This is because many patients with DKA/HHS develop tachypnea, which can affect the efficacy of an oral thermometer. In such cases, the rectal temperature is more sensitive for detecting fever. It should be noted, however, that some patients with severe infection or immunocompromised state-regardless of the presence or absence of tachypnea-may be normothermic or even hypothermic due to peripheral vasodilation-a poor prognostic sign. ${ }^{3}$ Blood and urine cultures should be obtained for all patients in whom infection is suspected.

Laboratory evaluation of patients with DKA may demonstrate a mild leukocytosis, a very common finding in DKA even in the absence of infection; leukocytosis is believed to result from elevated stress hormones such as cortisol and epinephrine. ${ }^{3}$

Infarction. Infarction is another important underlying cause of DKA/HHS and one that must not be overlooked. Screening for acute coronary syndrome through patient history, electrocardiography, and cardiac biomarkers should be performed in all patients older than age 40 years and in patients in whom there is any suggestion of myocardial ischemia.

A thorough neurological examination should be performed to assess for deficits indicative of stroke. Although neurological deficits can be due to severe hyperglycemia, most patients with focal neurological deficits from hyperglycemia also have altered mental status. Focal neurological deficits without a change in mental status are more likely to represent an actual stroke. ${ }^{4}$

Infant (Pregnancy). Pregnant patients are at an increased risk of developing DKA for several reasons, most notably due to the increased production of insulin-antagonistic hormones, which can lead to higher insulin resistance and thus increased insulin requirements during pregnancy. ${ }^{5}$ A pregnancy test is therefore indicated for all female patients of child-bearing age.

Indiscretion. Non-compliance with diet, such as taking in too many calories without appropriate insulin correction, and the ingestion of significant amounts of alcohol can lead to DKA. Eating disorders, particularly in young patients, may also contribute to recurrent cases. $^{3}$

[Lack of] Insulin. In insulin-dependent diabetes, skipped insulin doses or insulin pump failure can trigger DKA/HHS. In fact, missed insulin doses are becoming a more frequent cause of DKA than infections. ${ }^{1}$

Both DKA and HHS are usually triggered by an underlying illness or event. Therefore, clinicians should always focus on identifying precipitating causes such as acute infection, stroke, or myocardial infarction, as some require immediate treatment. In fact, DKA or HHS is rarely the primary cause of death; patients are much more likely to die from the precipitating event that caused DKA or HHS., ${ }^{1,3}$

\section{Assessing Disease Severity}

Mental status and $\mathrm{pH}$ and serum bicarbonate levels help clinicians determine the extent of disease severity, classifying patients as having mild, moderate, or severe DKA (Table 4). ${ }^{2}$ Patients at the highest risk for poor outcomes include those at the extremes of age, who have severe comorbidities, who have underlying infection, and who are hypotensive and/or in a comatose state. ${ }^{3}$

Patients who have HHS are much more likely to present with altered mental status, including coma. There is a linear relationship between osmolality and degree of altered mental status. Thus, diabetic patients with major changes in mental status but without high serum osmolality warrant immediate workup for alternative causes of their altered mental status. ${ }^{3}$ In addition, seizures, especially focal seizures, are relatively common in 


\section{DIABETIC KETOACIDOSIS AND HYPEROSMOLAR HYPERGLYCEMIC SYNDROME MANAGEMENT}

Table 4. Diabetic Ketoacidosis Severity

\begin{tabular}{llll}
\hline & Mild & Moderate & Severe \\
\hline $\mathrm{pH}$ & $7.25-7.30$ & $7.00-7.24$ & $<7.0$ \\
\hline Bicarbonate & $15-18 \mathrm{mEq} / \mathrm{L}$ & $10-15 \mathrm{mEq} / \mathrm{L}$ & $<10 \mathrm{mEq} / \mathrm{L}$ \\
\hline Mental Status & Alert & \pm Alert & Stupor or coma \\
\hline
\end{tabular}

Modified from Med Clin North Am. ${ }^{2}$

severe cases of HHS. Finally, highly abnormal blood pressure, pulse, and respiratory rate can also provide additional clues regarding the severity of hyperglycemic crisis.

Arterial Blood Gas Assessment: To Stick or Not to Stick?

In the past, measuring arterial blood gases (ABG) has been considered a mainstay in the evaluation of patients with DKA. But does an arterial stick, which is associated with some risk, really add essential information? One study by Ma et $\mathrm{al}^{6}$ evaluated whether ABG results significantly alter how physicians manage patients with DKA. In the study, the authors evaluated $200 \mathrm{ED}$ patients and found that ABG analysis only changed diagnosis in $1 \%$ of patients, altered treatment in $3.5 \%$ of patients, and changed disposition in only $1 \%$ of patients. Arterial stick partial pressure of oxygen and partial pressure of carbon dioxide altered treatment and disposition in only $1 \%$ of patients. Furthermore, the study results showed venous $\mathrm{pH}$ correlated very strongly with arterial $\mathrm{pH}(r=0.95){ }^{6}$ These findings demonstrate that ABG measurements rarely affect or alter DKA management, and support the use of venous $\mathrm{pH}$ as an adequate substitute for ABG testing.

\section{Euglycemia}

Euglycemic DKA has been reported in patients with type 1 diabetes who had been fasting or vomiting or who had received exogenous insulin prior to presentation. ${ }^{1}$ Euglycemia has also been reported in pregnant patients with type I diabetes. ${ }^{1}$ More recently, sodium glucose cotransporter 2 inhibitors (SGLT2) have been shown to cause euglycemic DKA. While the therapeutic mechanism of this drug class is to inhibit proximal tubular resorption of glucose, they can cause DKA by decreasing renal clearance of ketone bodies and increasing glucagon levels and promoting hepatic ketogenesis. Patients with DKA who are on SGLT2 inhibitors may present with only modestly elevated glucose levels (typically in the 200- to 300-mg/dL range), but have profound wide gap metabolic acidosis due to $\beta$-hydroxybutyrate acid and acetoacetate accumulation. ${ }^{7}$ When evaluating patients on SGLT2 inhibitors for DKA, EPs should not solely rely on glucose values but rather assess the patient's overall clinical picture, including the physical examination, vital signs, and $\mathrm{pH}$. Additionally, once resuscitation with intravenous (IV) fluids and insulin is initiated, it may take longer for patients who use SGLT2 inhibitors to clear ketoacids than patients with DKA who do not use these medications. ${ }^{8}$

\section{Treatment}

The goal of treatment for DKA and HHS is to correct volume deficits, hyperglycemia, and electrolyte abnormalities (Table 5). Three of the five therapies to manage DKA and HHS are mandatory: IV fluid resuscitation, IV insulin, and IV potassium. The other therapies, IV bicarbonate, and IV phosphate, should be considered, but are rarely required for DKA 


\section{DIABETIC KETOACIDOSIS AND HYPEROSMOLAR HYPERGLYCEMIC SYNDROME MANAGEMENT}

or HHS.

In general, management of HHS is less aggressive than that of DKA because HHS develops over a period of weeks-unlike DKA, which develops over only 1 to 2 days. Treatment of any underlying causes of HHS should occur simultaneously.

\section{Intravenous Fluids}

The goal of IV fluid therapy for patients with DKA is rehydration-not to "washout" ketones. Ketone elimination occurs through insulin-stimulated metabolism. When determining volume-replacement goals, it is helpful to keep in mind that patients with moderate-to-mild DKA typically have fluid deficiencies of 3 to $5 \mathrm{~L}$, and patients with severe DKA have fluid deficiencies between 5 and 6 L. Patients with HHS present with significantly higher fluid deficiencies of around 9 to $12 \mathrm{~L}$.

The initial goal of fluid management is to correct hypoperfusion with bolus fluids, followed by a more gradual repletion

Table 5. Five Treatments for Diabetic Ketoacidosis and/or Hyperosmolar Hyperglycemic State

Volume

Insulin

Potassium

Bicarbonate

Phosphate of remaining deficits. After bolus fluids are administered, the rate and type of subsequent IV fluid infusion varies depending on hemodynamics, hydration state, and serum sodium levels. ${ }^{2,3}$

Nonaggressive Vs Aggressive Fluid Management. One study by Adrogué et $\mathrm{al}^{9} \mathrm{compared} \mathrm{the}$ effects of managing DKA with aggressive vs nonaggressive fluid repletion. In the study, one group of patients received normal saline at 1,000 mL per hour for 4 hours, followed by normal saline at $500 \mathrm{~mL}$ per hour for 4 hours. The other group of patients received normal saline at a more modest rate of $500 \mathrm{~mL}$ per hour for 4 hours, followed by normal saline at $250 \mathrm{~mL}$ per hour for 4 hours. The authors found that patients in the less aggressive volume therapy group achieved a prompt and adequate recovery and maintained higher serum bicarbonate levels. ${ }^{9}$

Current recommendations for patients with DKA are to first treat patients with an initial bolus of $1,000 \mathrm{~mL}$ or $20 \mathrm{cc} / \mathrm{kg}$ of normal saline. Patients without profound dehydration should then receive $500 \mathrm{cc}$ of normal saline per hour for the first 4 hours of treatment, after which the flow rate may be reduced to 250 cc per hour. For patients with mild DKA, therapy can start at 250 cc per hour with a smaller bolus dose or no bolus dose. Patients with profound dehydration and poor perfusion, should receive crystalloid fluids wide open until perfusion has improved. Overall, volume resuscitation in HHS is similar to DKA. However, the EP should be cautious with respect to total fluid volume and infusion rates to avoid fluid overload, since many patients with HHS are elderly and may have congestive heart failure.

Crystalloid Fluid Type for Initial Resuscitation. Normal saline has been the traditional crystalloid fluid of choice for managing DKA and HHS. Recent studies, however, have shown some benefit to using balanced solutions (Ringer's lactate or PlasmaLyte) instead of normal saline. A recently published large study by Semler et $\mathrm{al}^{10}$ compared balanced crystalloid, in most cases lactated Ringers solution, to normal saline in critically ill adult patients, some of whom were diagnosed with DKA. The study demonstrated decreased mortality (from any cause) in the group who received balanced crystalloid fluid therapy 


\section{DIABETIC KETOACIDOSIS AND HYPEROSMOLAR HYPERGLYCEMIC SYNDROME MANAGEMENT}

and reduced need for renal-replacement therapy and reduced incidence of persistent renal dysfunction. The findings by Semler et $\mathrm{al}^{10}$ and findings from other smaller studies, calls into question whether normal saline is the best crystalloid to manage DKA and HHS. $^{11,12}$ It remains to be seen what modification the American Diabetes Association (ADA) will make to its current recommendations for fluid therapy, which were last updated in 2009.

It appears that though the use of Ringers lactate or PlasmaLyte to treat DKA usually raises a patient's serum bicarbonate level to $18 \mathrm{mEq} / \mathrm{L}$ at a more rapid rate than normal saline, the use of balanced solutions may result in longer time to lower blood glucose to $250 \mathrm{mg} / \mathrm{dL} .{ }^{13}$ However, by using a balanced solution, the hyperchloremic metabolic acidosis often seen with normal saline treatment will be avoided. ${ }^{12}$

Half-Normal Saline. An initially normal or increased serum sodium level, despite significant hyperglycemia, suggests a substantial free-water deficit. Calculating a corrected serum sodium can help quantify the degree of free-water deficit. ${ }^{3}$ While isotonic fluids remain the standard for initial volume load, clinicians should consider switching patients to half-normal saline following initial resuscitation if the corrected serum sodium is elevated above normal. The simplest estimation to correct sodium levels in DKA is to expect a decrease in sodium levels at a rate of at least $2 \mathrm{mEq} / \mathrm{L}$ per $100-\mathrm{mg} / \mathrm{dL}$ increase in glucose levels above $100 \mathrm{mg} / \mathrm{dL}$. For a more accurate calculation, providers can expect a drop in sodium of $1.6 \mathrm{mEq} / \mathrm{L}$ per $100 \mathrm{mg} / \mathrm{dL}$ increase of glucose up to a level of $400 \mathrm{mg} / \mathrm{dL}$ and then a fall of $2.4 \mathrm{mEq} / \mathrm{L}$ in sodium per every $100 \mathrm{mg} / \mathrm{dL}$ rise in glucose thereafter. ${ }^{14}$

Glucose. Patients with DKA require insulin therapy until ketoacidosis resolves. However, the average time to correct ketoacidosis from initiating treatment is about 12 hours compared to only 6 hours for correction of hyperglycemia. Since insulin therapy must be continued despite lower glucose levels, patients are at risk for developing hypoglycemia if glucose is not added to IV fluids. To prevent hypoglycemia and provide an energy source for ketone metabolism, patients should be switched to fluids containing dextrose when their serum glucose approaches 200 to $250 \mathrm{mg} / \mathrm{dL} \cdot{ }^{1,3}$ Typically, $5 \%$ dextrose in half-normal saline at 150 to $250 \mathrm{cc}$ per hour is usually adequate to achieve this goal.

Insulin

As previously noted, insulin therapy is required to treat hyperglycemic crises from DKA and HHS. In DKA there is an absolute insulin deficiency, whereas in HHS, there is a relative insulin deficiency. In HHS, there is not enough endogenous insulin to move glucose into the cells, but there is enough insulin to block a catabolic state. That is why the breakdown of fats and proteins does not occur, and why ketoacidosis and hyperkalemia are not seen in HHS. On the other hand, glucose elevations do occur, and are usually more extreme in HHS than DKA. There are five major therapeutic actions of insulin in DKA (Table 5), and it is imperative to determine serum potassium before starting an insulin infusion as insulin will drive potassium into the cell, worsening hypokalemia and promoting the development of life-threatening arrhythmias, including ventricular fibrillation, ventricular tachycardia, and torsades de pointes. The electrocardiogram does not accurately predict severity of hypokalemia and should not be used as a substitute for direct potassium measurement.

Loading Dose and Drip Rate. When treating adult patients with DKA or HHS, the ADA recommends an IV push loading dose of $0.1 \mathrm{U} / \mathrm{kg}$ insulin, followed by an hourly maintenance dose of $0.1 \mathrm{U} / \mathrm{kg}$. Alternatively, a continuous infusion of $0.16 \mathrm{U} / \mathrm{kg} / \mathrm{hr}$ can be used without a bolus. The rationale behind a bolus is the rapid saturation of insulin receptors, 


\section{DIABETIC KETOACIDOSIS AND HYPEROSMOLAR HYPERGLYCEMIC SYNDROME MANAGEMENT}

followed by a drip to maintain saturation of receptors. However, a recent prospective observational cohort study by Goyal et $\mathrm{al}^{15}$ questions the utility of the initial insulin bolus. The study compared DKA patients who received an initial insulin bolus to those who did not. Both groups were similar at baseline and received equivalent IV fluids and insulin drips. They found no statistically significant differences in the incidence of hypoglycemia, rate of serum glucose change, anion gap change, or length of stay in the ED or hospital. The authors concluded that administration of an insulin bolus has no significant benefit to patients and does not change clinically relevant end-points. ${ }^{15}$ At this time, there is no proven benefit to giving DKA patients an IV insulin bolus; moreover, doing so may further increase hypoglycemia. The use of an insulin bolus is particularly not recommended for use in pediatric patients with DKA due to a higher incidence of hypoglycemia in this patient population. ${ }^{16}$

As with DKA, the $\mathrm{ADA}^{3}$ recommends giving HHS patients an insulin bolus of $0.1 \mathrm{U} /$ $\mathrm{kg}$ followed by a continuous infusion at $0.1 \mathrm{U} / \mathrm{kg}$ per hour. It is crucial to monitor patients closely to ensure glucose levels do not fall too rapidly. Glucose levels should be kept between 150 to $200 \mathrm{mg} / \mathrm{dL}$ for patients with DKA and 200 to $300 \mathrm{mg} / \mathrm{dL}$ for patients with HHS until the conditions resolve; this may necessitate lowering the infusion rate to 0.02 to $0.05 \mathrm{U} / \mathrm{kg}$ per hour. In addition to frequent glucose monitoring, a basic metabolic panel and venous $\mathrm{pH}$ should be obtained every 2 to 4 hours while a patient is on an insulin drip. ${ }^{3}$

Subcutaneous Vs Intravenous Insulin for DKA. Several small studies evaluating patients with mild-moderate DKA demonstrated similar outcomes when subcutaneous (SQ) insulin was used instead of IV insulin. However, SQ injections require more frequent dosing (every 1 to 2 hours) and still require close monitoring of blood glucose. This monitoring frequency is usually not feasible on a hospital floor, but may be feasible on step-down units, thus avoiding admission to the intensive care unit (ICU) for patients who do not otherwise require ICU-level of care. ${ }^{17}$ Subcutaneous insulin should not be given to patients with severe acidosis, hypotension, or altered mental status. The ADA consensus statement continues to recommend IV infusion of regular insulin as the preferred route due to its short half-life and easy titration. ${ }^{3}$

Determining When to Switch to Subcutaneous Insulin. Ideally, patients are not in the ED long enough to have their metabolic abnormalities corrected, as this usually requires several hours. In DKA, the insulin drip should continue until the blood glucose is less than 200 and at least two of the following conditions are met: the anion gap is less than 12, venous $\mathrm{pH}$ greater than 7.3, and serum bicarbonate $>15$. In HHS, osmolality and mental status should both return to normal prior to stopping the infusion. In both cases, subcutaneous insulin should be administered at least 1 to 2 hours before stopping the drip to prevent recurrent crisis. ${ }^{1,3}$

Refractory Acidosis. First and foremost, refractory acidosis should prompt a diligent source for dead gut, abscess, and underlying sepsis. While vomiting and diffuse abdominal pain are common in DKA and are related to ketoacidosis, these symptoms are atypical of HHS and should raise suspicion for underlying pathology. ${ }^{3}$ Additionally, a lower than expected bicarbonate level can also occur from resuscitation with large volumes of normal saline, resulting in a hyperchloremic non-gap metabolic acidosis.

\section{Potassium}

Both DKA and HHS patients have total body potassium deficits due to osmotic diuresis that require careful repletion. Deficits can be substantial: The average total whole body potassium deficit in DKA is 3 to $5 \mathrm{mEq} / \mathrm{kg}$. ${ }^{2}$ Clinicians should exercise caution, however, 


\section{DIABETIC KETOACIDOSIS AND HYPEROSMOLAR HYPERGLYCEMIC SYNDROME MANAGEMENT}

Table 6. Bicarbonate Management for Diabetic Ketoacidosis and Hyperosmolar Hyperglycemic State

\begin{tabular}{ll}
\hline Potential Benefits & Potential Risks \\
\hline Reverses acidosis & Intracellular acidosis \\
\hline Improves cardiac output & Increased calcium, hydrogen, potassium fluxes \\
\hline Increases fibrillatory threshold & Hypokalemia, tissue hypoxia \\
\hline Improves insulin sensitivity & Hyperosmolarity, hypernatremia \\
\hline Decreased work of breathing & Increased carbon dioxide generation, respiratory acidosis \\
\hline Decreased length of coma & Paradoxical cerebral spinal fluid acidosis \\
\hline
\end{tabular}

since DKA patients may be hyperkalemic initially despite a total body potassium deficit. Early hyperkalemia is due to the transmembrane shift of potassium secondary to acidosis and insulin deficiency as well as hypertonicity. If initial potassium is greater than 5.2 $\mathrm{mEq} / \mathrm{L}$, potassium should not be administered but instead rechecked in 1 to 2 hours. If the potassium level is 4.0 to $5.2 \mathrm{mEq} / \mathrm{L}$, then $10 \mathrm{mEq}$ per hour is usually adequate. For levels between 3.3 and $4.0 \mathrm{mEq} / \mathrm{L}$, administer potassium chloride at $20 \mathrm{mEq}$ per hour. For levels less than $3.3 \mathrm{mEq} / \mathrm{L}$, insulin should be held and potassium chloride should be aggressively repleted at 20 to $30 \mathrm{mEq}$ per hour with continuous cardiac monitoring. ${ }^{1-3}$ Failure to recognize and act on critical potassium levels is a known cause of unexpected death in DKA. During the first hour of DKA onset, patients are more likely to die from hyperkalemia. Later, while the patient is "stabilizing" on an insulin infusion, potassium levels will fall as insulin drives potassium back into cells.

\section{Bicarbonate}

Bicarbonate has many theoretical benefits but also has potential risks (Table 6). In general, bicarbonate should not be given to patients whose $\mathrm{pH}$ is above 6.9. Bicarbonate may provide some benefits to patients who have a $\mathrm{pH}$ below 6.9 , but only in certain situations. Clinicians must always keep in mind that IV bicarbonate can cause a paradoxical respiratory acidosis in the central nervous system. ${ }^{2}$ When administered, bicarbonate increases serum bicarbonate which allows the $\mathrm{pH}$ to rise as the acidosis decreases, in turn decreasing the need for hyperventilation, with a resultant rise in carbon dioxide levels. Because carbon dioxide can freely diffuse across the blood brain barrier but bicarbonate cannot, carbon dioxide levels rise in the cerebral spinal fluid (CSF), but there are no acute changes in CSF bicarbonate values. The result is an increased CSF carbon dioxide and an unchanged CSF bicarbonate value, resulting in an acute CSF respiratory acidosis. Additionally, bicarbonate has a very high osmolarity and is very hypernatremic relative to sera. Because of these potential deleterious effects of bicarbonate, we only recommend the rapid infusion of bicarbonate in DKA for hyperkalemic emergencies and impending cardiopulmonary arrest. Profoundly acidotic patients with $\mathrm{pH}$ values below 6.9 who are not improving with early aggressive care can potentially benefit from the careful administration of bicarbonate given relatively slowly. ${ }^{2}$ It is critically important that the EP recognizes that bicarbonate administration is the only therapeutic variable associated with cerebral edema in children with DKA and is usually seen within 4 to 12 hours after treatment. ${ }^{18}$ 


\section{DIABETIC KETOACIDOSIS AND HYPEROSMOLAR HYPERGLYCEMIC SYNDROME MANAGEMENT}

Table 7. Hyperosmolar Hyperglycemic State: Clinical Pearls

Find the underlying etiology

Treat carefully to avoid volume overload

Much higher mortality rate compared to diabetic ketoacidosis

Table 8. Diabetic Ketoacidosis: Clinical Pearls

Treat patients with normal saline prior to starting insulin therapy

Administer a fluid bolus to patients prior to giving

Sodium/glucose cotransporter-2 inhibitors: glucose levels intravenous saline infusion within normal limits, $\mathrm{pH}$ low

Beware of "normal" sodium levels

Bicarbonate is hyperosmolar and hypercarbic

Check patients' potassium levels before starting patients on insulin

Replete phosphate only if phosphate level is $<1-1.5$

Venous $\mathrm{pH}$, not arterial blood gas

Look for precipitating cause

Phosphate

Since there is no proven benefit to giving phosphate to adult patients with DKA, it is rarely used, except in specific situations other than pediatric DKA. Similar to potassium, initial serum phosphate levels do not reflect total body phosphate levels due to transmembrane shifts. ${ }^{2,3}$ Phosphate repletion is most beneficial for patients who have cachexia, respiratory depression, anemia, cardiac dysfunction, or phosphate values lower than 1.0 to 1.5. If given, 20 to $30 \mathrm{mEq} / \mathrm{L}$ potassium phosphate $\left(\mathrm{K}_{2} \mathrm{PO}_{4}\right)$ added to fluids is usually sufficient. ${ }^{3}$ Overly aggressive phosphate administration $(>4.5 \mathrm{mmol} / \mathrm{h}$ or 1.5 $\mathrm{mL} / \mathrm{h}$ potassium phosphate) can cause severe hypocalcemia and should be avoided. ${ }^{1,3}$ In pediatric patients, up to one-half of potassium requirements are often given as potassium phosphate, but this may vary by institution.

\section{Conclusion}

Both DKA and HHS are diabetic emergencies that must be approached and managed systematically to correct underlying dehydration and metabolic abnormalities. Patient care begins by determining the etiology of these conditions, especially HHS. Once the cause has been identified, patients should be treated with bolus fluids to obtain adequate perfusion, followed by IV fluid infusion. Clinicians should carefully monitor the serum sodium level of patients with DKA or HHS to determine the ideal amount and type of fluid required, and also should measure potassium levels prior to starting patients on insulin. (Tables 7 and 8 summarize important clinical pearls when treating patients with DKA or HHS.) 


\section{References}

1. Nyenwe EA, Kitabchi AE. The evolution of diabetic ketoacidosis: an update of its etiology, pathogenesis and management. Metabolism. 2016;65(4):507-521. doi:10.1016/j.metabol.2015.12.007.

2. Fayfman M, Pasquel FJ, Umpierrez GE. Management of hyperglycemic crises: diabetic ketoacidosis and hyperglycemic hyperosmolar state. Med Clin North Am. 2017;101(3):587-606. doi:10.1016/j.mcna.2016.12.011.

3. Kitabchi AE, Umpierrez GE, Miles JM, Fisher JN. Hyperglycemic crises in adult patients with diabetes. Diabetes Care. 2009;32(7):1335-1343. doi:10.2337/dc09-9032.

4. Fugate JE, Rabinstein AA. Absolute and relative contraindications to IV rt-PA for acute ischemic stroke. Neurohospitalist. 2015;5(3):110-121. doi:10.1177/1941874415578532.

5. Kamalakannan D, Baskar V, Barton DM, Abdu TA. Diabetic ketoacidosis in pregnancy. Postgrad Med J. 2003;79(9):454-457.

6. Ma OJ, Rush MD, Godfrey MM, Gaddis G. Arterial blood gas results rarely influence emergency physician management of patients with suspected diabetic ketoacidosis. Acad Emerg Med. 2003;10(8):836-841.

7. Taylor S, Blau J, Rother K. SGLT2 Inhibitors may predispose to ketoacidosis. J Clin Endocrinol Metab. 2015;100(8):2849-2852. doi:10.1210/jc.2015-1884.

8. Kum-Nji JS, Gosmanov AR, Steinberg H, Dagogo-Jack S. Hyperglycemic, high anion-gap metabolic acidosis in patients receiving SGLT-2 inhibitors for diabetes management. J Diabetes Complications. 2017;31(3):611-614. doi:10.1016/j.jdiacomp.2016.11.004.

9. Adrogué HJ, Barrero J, Eknoyan G. Salutary effects of modest fluid replacement in the treatment of adults with diabetic ketoacidosis. Use in patients without extreme volume deficit. JAMA. 1989;262(15):2108-2013.

10. Semler MW, Self WH, Wanderer JP, et al; SMART Investigators and the Pragmatic Critical Care Research Group. Balanced crystalloid versus saline in critically ill adults. N Engl J Med. 2018;378(9):829-839. doi:10.1056/NEJMoa1711584

11. Chua HR, Venkatesh B, Stachowski E, et al. Plasma-Lyte 148 vs $0.9 \%$ saline for fluid resuscitation in diabetic ketoacidosis. J Crit Care. 2012;27(2):138-145. doi:10.1016/j.jcrc.2012.01.007.

12. Mahler S, Conrad S, Wang H, Arnold T. Resuscitation with balanced electrolyte solution prevents hyperchloremic metabolic acidosis in patients with diabetic ketoacidosis. Am J Emerg Med. 2011;29(9):1194-1197. doi:10.1016/j. ajem.2010.07.015.

11. Van Zyl DG, Rheeder P, Delport E. Fluid management in diabetic-acidosis--Ringer's lactate versus normal saline: a randomized controlled trial. QJM. 2012;105(4):337-343. doi:10.1093/qjmed/hcr226.

14. Penne EL, Thijssen S, Raimann JG, Levin NW, Kotanko P. Correction of serum sodium for glucose concentration in hemodialysis patients with poor glucose control. Diabetes Care. 2010;33(7):e91. doi:10.2337/dc10-0557.

15. Goyal N, Miller JB, Sankey SS, Mossallam U. Utility of initial bolus insulin in the treatment of diabetic ketoacidosis. J Emerg Med. 2010;38(4):422-427. doi:10.1016/j.jemermed.2007.11.033.

16. Wolfsdorf JI, Allgrove J, Craig ME, et al; International Society for Pediatric and Adolescent Diabetes. Diabetic ketoacidosis and hyperglycemic hyperosmolar state. Pediatr Diabetes. 2014;15(Suppl 20):154-179. doi:10.1111/ pedi.12165.

17. Cohn BG, Keim SM, Watkins JW, Camargo CA. Does management of diabetic ketoacidosis with subcutaneous rapid-acting insulin reduce the need for intensive care unit admission? J Emerg Med. 2015;49(4):530-538. doi:10.1016/j.jemermed.2015.05.016.

18. Glaser N, Barnett P, McCaslin I, et al; Pediatric Emergency Medicine Collaborative Research Committee of the American Academy of Pediatrics. Risk factors for cerebral edema in children with diabetic ketoacidosis. The Pediatric Emergency Medicine Collaborative Research Committee of the American Academy of Pediatrics. N Engl Med. 2001;344(4):264-269. doi:10.1056/NEJM200101253440404. 